\title{
On a general class of weakly Picard operators
}

\author{
I. Altun, H. A. Hançer, and G. Minak
}




\title{
ON A GENERAL CLASS OF WEAKLY PICARD OPERATORS
}

\author{
I. ALTUN, H. A. HANÇER, AND G. MINAK
}

Received 15 March, 2014

\begin{abstract}
In this paper we show that on complete metric spaces the class of weakly Picard operators contains some operators which are more general than the class of almost contractions.

2010 Mathematics Subject Classification: 54H25, 47H10

Keywords: Fixed point, Picard operator, weakly Picard operator, almost contraction, almost $\theta$ -
\end{abstract} contraction.

\section{InTRODUCTION AND PRELIMINARIES}

One of the most important concepts of fixed point theory is the Picard operator. We know that a self mapping $T$ of a nonempty set $X$ is Picard operator, if $T$ has a unique fixed point and the sequence of successive approximation for any initial point converges to this unique fixed point. Therefore, lot of different types of contractions are Picard operators. (See for more details [1-4]). On the other hand, Rus [6] introduced the concept of weakly Picard operator: a self mapping $T$ of a nonempty set $X$ is weakly Picard operator, if the set of fixed points of $T$ is nonempty and the sequence of successive approximation for any initial point converges to a fixed point of $T$. There exist many weakly Picard operators in the literature, but one of the most interesting of them is the class of almost contraction, which was introduced by Berinde [2], on complete metric space. Berinde [3] shows that the concept of almost contraction is more general than the lot of different types of contractions in the literature.

For the sake of completeness we recall the concept of almost contraction and some related results.

Let $(X, d)$ be a metric space and $T: X \rightarrow X$ be a map. $T$ is said to be an almost contraction if there exist a constant $\delta \in(0,1)$ and some $L \geq 0$ such that

$$
d(T x, T y) \leq \delta d(x, y)+L d(y, T x)
$$

for all $x, y \in X$. 
Note that, by the symmetry property of the distance, the almost contractive condition implicitly includes the following dual one

$$
d(T x, T y) \leq \delta d(x, y)+L d(x, T y)
$$

for all $x, y \in X$. So, in order to check the almost contractiveness of a mapping $T$, it is necessary to check both (1.1) and (1.2).

Theorem 1 ([3]). Every almost contraction on complete metric space is a weakly Picard operator.

On the other hand, a new type of contractive maps has been introduced by Jleli and Samet [5]. Throughout this study, we called it as $\theta$-contraction.

Let $\Theta$ be the set of all functions $\theta:(0, \infty) \rightarrow(1, \infty)$ satisfying the following conditions:

$\left(\Theta_{1}\right) \theta$ is nondecreasing,

$\left(\Theta_{2}\right)$ for each sequence $\left\{t_{n}\right\} \subset(0, \infty), \lim _{n \rightarrow \infty} \theta\left(t_{n}\right)=1$ if and only if $\lim _{n \rightarrow \infty} t_{n}=0^{+}$,

$\left(\Theta_{3}\right)$ there exist $r \in(0,1)$ and $l \in(0, \infty]$ such that $\lim _{t \rightarrow 0^{+}} \frac{\theta(t)-1}{t^{r}}=l$.

Definition 1 ([5]). Let $(X, d)$ be a metric space and $T: X \rightarrow X$ be a mapping. Given $\theta \in \Theta$, we say that $T$ is $\theta$-contraction if there exists $k \in(0,1)$ such that

$$
\theta(d(T x, T y)) \leq[\theta(d(x, y))]^{k} .
$$

for all $x, y \in X$ with $d(T x, T y)>0$.

If we consider the different type of mapping $\theta$ in Definition 1, we obtain some of variety of contractions. For example, let $\theta:(0, \infty) \rightarrow(1, \infty)$ be given by $\theta(t)=e^{\sqrt{t}}$. It is clear that $\theta \in \Theta$. Then (1.3) turns to

$$
d(T x, T y) \leq k^{2} d(x, y) \text {, for all } x, y \in X, T x \neq T y .
$$

It is clear that for $x, y \in X$ such that $T x=T y$ the inequality $d(T x, T y)$ $\leq k^{2} d(x, y)$ also holds. Therefore $T$ is an ordinary contraction. Similarly, let $\theta$ : $(0, \infty) \rightarrow(1, \infty)$ be given by $\theta(t)=e^{\sqrt{t e^{t}}}$. It is clear that $\theta \in \Theta$. Then (1.3) turns to

$$
\frac{d(T x, T y)}{d(x, y)} e^{d(T x, T y)-d(x, y)} \leq k^{2} \text {, for all } x, y \in X, T x \neq T y .
$$

In addition, we have concluded that every $\theta$-contraction $T$ is a contractive mapping, i.e.,

$$
d(T x, T y)<d(x, y) \text {, for all } x, y \in X, T x \neq T y .
$$

Thus, every $\theta$-contraction is a continuous mapping. On the other side, Example in [5] shows that the mapping $T$ is not ordinary contraction but, but it is a $\theta$-contraction with $\theta(t)=e^{\sqrt{t e^{t}}}$. Thus, the following theorem, which was given as a corollary by Jleli and Samet [5], is a proper generalization of Banach Contraction Principle. 
Theorem 2 (Corollary 2.1 of [5]). Let $(X, d)$ be a complete metric space and $T: X \rightarrow X$ be a $\theta$-contraction. Then $T$ has a unique fixed point in $X$.

If we examine the proof of the above theorem, we can say that every $\theta$-contraction on complete metric space is a Picard operator.

In the next section, we introduce a new concept of almost $\theta$-contraction on metric spaces. Then we show that the class of almost $\theta$-contractions are more general than the class of almost contractions and we also show that they are still weakly Picard operators on complete metric spaces.

\section{MAIN RESULT}

Definition 2. Let $(X, d)$ be a metric space and $T: X \rightarrow X$ be a mapping. Given $\theta \in \Theta$, we say that $T$ is almost $\theta$-contraction if there exist $k \in(0,1)$ and $\lambda \geq 0$ such that, for all $x, y \in X$ with $d(T x, T y)>0$

$$
\theta(d(T x, T y)) \leq[\theta(d(x, y)+\lambda d(y, T x))]^{k} .
$$

Note that, by the symmetry property of the distance, the almost $\theta$-contractive condition implicitly includes the following dual one

$$
\theta(d(T x, T y)) \leq[\theta(d(x, y)+\lambda d(x, T y))]^{k}
$$

for all $x, y \in X$ with $d(T x, T y)>0$. So, in order to check the almost

$\theta$-contractiveness of a mapping $T$, it is necessary to check both (2.1) and (2.2).

By the considering $\theta(\alpha)=e^{\sqrt{t}}$, we can say that every almost contraction is also almost $\theta$-contraction.

Our main result is as follows:

Theorem 3. Every almost $\theta$-contraction on complete metric space is a weakly Picard operator.

Proof. Let $(X, d)$ be a complete metric space and $T: X \rightarrow X$ be an almost $\theta$ contraction. Let $x_{0} \in X$ be an arbitrary point in $X$. Define a sequence $\left\{x_{n}\right\}$ by $x_{n}=T^{n} x_{0}$ for all $n \in \mathbb{N}$. If there exists $n_{0} \in \mathbb{N}$ such that $x_{n_{0}}=x_{n_{0}+1}$, then $x_{n_{0}}$ is a fixed point of $T$. So we can suppose that $x_{n} \neq x_{n+1}$ for all $n \in \mathbb{N}$. Now, from (2.1), for all $n \in \mathbb{N}$, we have

$$
\begin{aligned}
\theta\left(d\left(x_{n}, x_{n+1}\right)\right) & =\theta\left(d\left(T x_{n-1}, T x_{n}\right)\right) \\
& \leq\left[\theta\left(d\left(x_{n-1}, x_{n}\right)+\lambda d\left(x_{n}, T x_{n-1}\right)\right)\right]^{k} \\
& =\left[\theta\left(d\left(x_{n-1}, x_{n}\right)\right]^{k} .\right.
\end{aligned}
$$

Therefore we have

$$
1<\theta\left(d\left(x_{n}, x_{n+1}\right)\right) \leq\left[\theta\left(d\left(x_{0}, x_{1}\right)\right]^{k^{n}}\right.
$$


for all $n \in \mathbb{N}$. Letting $n \rightarrow \infty$ in (2.3), we obtain

$$
\lim _{n \rightarrow \infty} \theta\left(d\left(x_{n}, x_{n+1}\right)\right)=1
$$

which implies from $\left(\Theta_{2}\right)$ that

$$
\lim _{n \rightarrow \infty} d\left(x_{n}, x_{n+1}\right)=0^{+} .
$$

Therefore from $\left(\Theta_{3}\right)$ there exist $r \in(0,1)$ and $l \in(0, \infty]$ such that

$$
\lim _{n \rightarrow \infty} \frac{\theta\left(d\left(x_{n}, x_{n+1}\right)\right)-1}{\left[d\left(x_{n}, x_{n+1}\right)\right]^{r}}=l .
$$

As in the proof of Theorem 2.1 in [5], we can obtain

$$
\lim _{n \rightarrow \infty} n\left[d\left(x_{n}, x_{n+1}\right)\right]^{r}=0 .
$$

Thus, there exits $n_{1} \in \mathbb{N}$ such that $n\left[d\left(x_{n}, x_{n+1}\right)\right]^{r} \leq 1$ for all $n \geq n_{1}$. So, we have, for all $n \geq n_{1}$

$$
d\left(x_{n}, x_{n+1}\right) \leq \frac{1}{n^{1 / r}} .
$$

Let $m>n>n_{1}$, then considering (2.5), we have

$$
\begin{aligned}
d\left(x_{n}, x_{m}\right) & \leq d\left(x_{n}, x_{n+1}\right)+d\left(x_{n+1}, x_{n+2}\right)+\cdots+d\left(x_{m-1}, x_{m}\right) \\
& =\sum_{i=n}^{m-1} d\left(x_{i}, x_{i+1}\right) \leq \sum_{i=n}^{m-1} \frac{1}{i^{1 / r}} \leq \sum_{i=n}^{\infty} \frac{1}{i^{1 / r}} .
\end{aligned}
$$

Since the series $\sum_{i=1}^{\infty} \frac{1}{i^{1 / r}}$ is convergent, we get $d\left(x_{n}, x_{m}\right) \rightarrow 0$. This yields that $\left\{x_{n}\right\}$ is a Cauchy sequence in $(X, d)$. Since $(X, d)$ is a complete metric space, there exists $z \in X$ such that $\lim _{n \rightarrow \infty} x_{n}=z$.

On the other hand, from $\left(\Theta_{1}\right)$ and (2.1), it is easy to conclude that

$$
d(T x, T y)<d(x, y)+\lambda d(y, T x)
$$

for all $x, y \in X$ with $T x \neq T y$. Therefore, for all $x, y \in X$

$$
d(T x, T y) \leq d(x, y)+\lambda d(y, T x)
$$

is satisfied. Thus, from (2.6),

$$
\begin{aligned}
d\left(x_{n+1}, T z\right) & =d\left(T x_{n}, T z\right) \\
& \leq d\left(x_{n}, z\right)+\lambda d\left(z, x_{n+1}\right) .
\end{aligned}
$$

Letting the $n \rightarrow \infty$ in the above inequality, we have $d(z, T z)=0$ and so $z=T z$. Therefore, $T$ is a weakly Picard operator on $X$.

In the following example, the mapping $T$ is not almost contraction, but it is almost $\theta$-contraction on a complete metric space and so is weakly Picard operator. 
Example 1. Consider the complete metric space $(X, d)$, where $X=\{0,1,2, \cdots\}$ and $d: X \times X \rightarrow[0, \infty)$ is given by

$$
d(x, y)=\left\{\begin{array}{cc}
0 & , \quad x=y \\
x+y & , \quad x \neq y
\end{array} .\right.
$$

Let $T: X \rightarrow X$ be defined by

$$
T x=\left\{\begin{array}{cc}
0, & x \in\{0,1\} \\
x-1, & x \geq 2
\end{array} .\right.
$$

Then $T$ is not almost contraction. Indeed, for $y \geq 2$ and $x=y+1$, then we have $d(y, T x)=0$ and

$$
\sup \left\{\frac{d(T x, T y)}{d(x, y)}: x, y \in X, x=y+1\right\}=1 .
$$

Thus, we can not find $\delta \in(0,1)$ and $L \geq 0$ satisfying (1.1). Therefore, using Theorem 1 we can not decide that whether $T$ is weakly Picard operator.

Now, we claim that $T$ is almost $\theta$-contraction with $\theta(t)=e^{\sqrt{t e^{t}}}, k=e^{-\frac{1}{2}}$ and $\lambda \geq 0$. To see this we have to show that

$$
\theta(d(T x, T y)) \leq[\theta(d(x, y)+\lambda d(y, T x))]^{k}
$$

for all $x, y \in X$ with $d(T x, T y)>0$. For this, it is sufficient to show

$$
\theta(d(T x, T y)) \leq[\theta(d(x, y))]^{k}
$$

or equivalently

$$
\frac{d(T x, T y)}{d(x, y)} e^{d(T x, T y)-d(x, y)} \leq e^{-1} .
$$

for all $x, y \in X$ with $d(T x, T y)>0$. First, observe that

$$
d(T x, T y)>0 \Leftrightarrow \text { the set }\{x, y\} \cap\{0,1\} \text { is singleton or empty. }
$$

Since (2.7) is symmetric with respect to $x$ and $y$, we may assume $x>y$ in the following cases.

Case 1. Let $\{x, y\} \cap\{0,1\}$ is singleton. Then $d(T x, T y)=x-1$ and $d(x, y) \leq$ $\max \{x, x+1\}$, and so we have

$$
\frac{d(T x, T y)}{d(x, y)} e^{d(T x, T y)-d(x, y)} \leq \frac{x-1}{x} e^{-1} \leq e^{-1} .
$$

Case 2. Let $\{x, y\} \cap\{0,1\}$ is empty. Then $d(T x, T y)=x+y-2$ and $d(x, y)=$ $x+y$ and so we have

$$
\frac{d(T x, T y)}{d(x, y)} e^{d(T x, T y)-d(x, y)}=\frac{x+y-2}{x+y} e^{-2} \leq e^{-2} \leq e^{-1} .
$$


This shows that $T$ is almost $\theta$-contraction with with $\theta(t)=e^{\sqrt{t e^{t}}}$ and so by Theorem $3, T$ is a weakly Picard operator.

Now we give some examples showing that $T$ is almost $\theta$-contraction but not $\theta$ contraction.

Example 2. Let $X=[0,1] \cup\{2,3\}$ and $d(x, y)=|x-y|$, then $(X, d)$ is complete metric space. Define a map $T: X \rightarrow X$,

$$
T x=\left\{\begin{array}{cc}
\frac{1-x}{2}, & x \in[0,1] \\
x & , \quad x \in\{2,3\} .
\end{array} .\right.
$$

Since $d(T 2, T 3)=1=d(2,3)$, then for all $\theta \in \Theta$ and $k \in(0,1)$ we have

$$
\theta(d(T 2, T 3))=\theta(1)>[\theta(1)]^{k}=[\theta(d(2,3))]^{k} .
$$

Therefore, $T$ is not $\theta$-contraction. That is, Theorem 2 can not be applied to this example.

Now, we claim that $T$ is almost $\theta$-contraction with $\theta(t)=e^{\sqrt{t}}, k=\frac{1}{\sqrt{2}}$ and $\lambda=4$. To see this have to show that

$$
\theta(d(T x, T y)) \leq[\theta(d(x, y)+\lambda d(y, T x))]^{k}
$$

for all $x, y \in X$ with $d(T x, T y)>0$ or equivalently

$$
d(T x, T y) \leq \frac{1}{2} d(x, y)+2 d(y, T x)
$$

for all $x, y \in X$ with $x \neq y$.

Now we consider the following cases:

Case 1. Let $x, y \in[0,1]$, then

$$
\begin{aligned}
d(T x, T y) & =\frac{1}{2}|x-y|=\frac{1}{2} d(x, y) \\
& \leq \frac{1}{2} d(x, y)+2 d(y, T x),
\end{aligned}
$$

that is, (2.8) is satisfied.

Case 2. Let $x, y \in\{2,3\}$, then

$$
\begin{aligned}
d(T x, T y) & =|x-y| \leq \frac{5}{2}|x-y| \\
& =\frac{1}{2} d(x, y)+2 d(y, T x),
\end{aligned}
$$

that is, (2.8) is satisfied.

Case 3. Let $x \in[0,1]$ and $y \in\{2,3\}$, then

$$
d(T x, T y)=d(y, T x)
$$




$$
\leq \frac{1}{2} d(x, y)+2 d(y, T x)
$$

that is, (2.8) is satisfied.

Case 4. Let $x \in\{2,3\}$ and $y \in[0,1]$, then

$$
\begin{aligned}
d(T x, T y) & =\left|\frac{2 x+y-1}{2}\right|=\frac{2 x+y-1}{2} \\
& \leq \frac{5}{2}(x-y) \\
& =\frac{1}{2} d(x, y)+2 d(y, T x),
\end{aligned}
$$

that is, (2.8) is satisfied.

Therefore $T$ is almost $\theta$-contraction with $\theta(t)=e^{\sqrt{t}}$ and so it is a weakly Picard operator.

Example 3. Consider the complete metric space $(X, d)$, where $X=\{1,3,5, \cdots\} \cup\{2\}$ and $d(x, y)=|x-y|$. Let $T: X \rightarrow X$ be defined by

$$
T x=\left\{\begin{array}{cc}
3, & x \in\{2,3\} \\
1, & \text { otherwise }
\end{array} .\right.
$$

Then it is easy to see that $T$ is not $\theta$-contraction. But $T$ is almost $\theta$-contraction with $k=\frac{1}{\sqrt{2}}, \lambda \geq 3$ and $\theta(t)=e^{\sqrt{t}}$.

In the following theorem, by adding an extra condition on almost $\theta$-contractions on complete metric space, we show that they are also Picard operators.

Theorem 4. Let $(X, d)$ be a complete metric space and $T: X \rightarrow X$ be an almost $\theta$ contraction. Suppose that $T$ also satisfies the following condition: there exist $\theta_{1} \in \Theta$ and some $\lambda_{1} \geq 0$ and $k_{1} \in(0,1)$ such that

$$
\theta_{1}(d(T x, T y)) \leq\left[\theta_{1}\left(d(x, y)+\lambda_{1} d(x, T x)\right)\right]^{k_{1}}
$$

for all $x, y \in X$ with $d(T x, T y)>0$. Then $T$ is a Picard operator.

Proof. Suppose that, there are two fixed point $z$ and $w$ of $T$. If $d(z, w)=0$, it is clear that $z=w$. Assume that $d(z, w)>0$. By (2.9) with $x=z$ and $y=w$, we have

$$
\begin{aligned}
\theta_{1}(d(z, w)) & =\theta_{1}(d(T z, T w)) \\
& \leq\left[\theta_{1}\left(d(z, w)+\lambda_{1} d(z, T z)\right)\right]^{k_{1}} \\
& =\left[\theta_{1}(d(z, w))\right]^{k_{1}},
\end{aligned}
$$

which is a contradiction. Therefore $T$ has a unique fixed point and so it is a Picard operator. 


\section{ACKNOWLEDGEMENT}

The authors are grateful to the referees because their suggestions contributed to the improvement of the paper.

\section{REFERENCES}

[1] V. Berinde, "Approximating fixed points of weak $\varphi$-contractions using the Picard iteration," Fixed Point Theory, vol. 4, no. 2, pp. 131-147, 2003.

[2] V. Berinde, "On the approximation of fixed points of weak contractive mappings," Carpathian J. Math., vol. 19, no. 1, pp. 7-22, 2003.

[3] V. Berinde, "Approximating fixed points of weak contractions using the Picard iteration," Nonlinear Analysis Forum, vol. 9, no. 1, pp. 43-53, 2004.

[4] V. Berinde, Iterative approximation of fixed points, 2nd ed., ser. Lecture Notes in Mathematics. Berlin: Springer, 2007, vol. 1912.

[5] M. Jleli and B. Samet, "A new generalization of the Banach contraction principle," J. Inequal. Appl., vol. 2014, no. 38, pp. 1-8, 2014.

[6] I. A. Rus, "Weakly Picard mappings," Comment. Math. Univ. Carolin., vol. 34, no. 4, pp. 769-773, 1993.

\section{Authors' addresses}

\section{Altun}

Department of Mathematics, Faculty of Science and Arts, Kirikkale University, 71450 Yahsihan, Kirikkale, Turkey

E-mail address: ishakaltun@yahoo.com

\section{H. A. Hançer}

Department of Mathematics, Faculty of Science and Arts, Kirikkale University, 71450 Yahsihan, Kirikkale, Turkey

E-mail address: haticeaslanhancer@yahoo.com

\section{G. Minak}

Department of Mathematics, Faculty of Science and Arts, Kirikkale University, 71450 Yahsihan, Kirikkale, Turkey

E-mail address: g.minak.28@gmail.com 